Open J. Math. Sci., Vol. 2(2018), No. 1, pp. 84 - 92

Website: https://pisrt.org/psr-press/journals/oms/

ISSN: 2523-0212 (Online) 2616-4906 (Print)

http://dx.doi.org/10.30538/oms2018.0019

\title{
ON A CLASS OF NEW HYPERGEOMETRIC TRANSFORMATIONS
}

\author{
GIOVANNI MINGARI SCARPELLO, DANIELE RITELLI ${ }^{1}$
}

\begin{abstract}
In this article we continue the investigations presented in our previous papers $[1,2,3,4]$, presenting some, for the best of our knowledge, new transformations of the Gauss hypergeometric function (4) and (13). They have been obtained using only elementary methods and stem from a couple of integrals evaluated in terms of complete elliptic integral of first kind by Legendre in [5] Chapter XXVII, at sections II and III.
\end{abstract}

Mathematics Subject Classification: 33E05, 33C05.

Key words and phrases: hypergeometric function; complete elliptic integral; hypergeometric transformations; modular transformations.

\section{Introduction}

Hypergeometric transformations are undoubtedly a fascinating field of investigation, which originates from the identities of Pfaff (1) and Euler (2)

$$
\begin{aligned}
& { }_{2} \mathrm{~F}_{1}\left(\begin{array}{c|c}
a ; b & x \\
c & x
\end{array}\right)=(1-x)^{-a}{ }_{2} \mathrm{~F}_{1}\left(\begin{array}{c|c}
a ; c-b & x \\
c & x-1
\end{array}\right), \\
& { }_{2} \mathrm{~F}_{1}\left(\begin{array}{c|c}
a ; b & x \\
c &
\end{array}\right)=(1-x)^{c-a-b}{ }_{2} \mathrm{~F}_{1}\left(\begin{array}{c|c}
c-a ; c-b & x \\
c & x
\end{array}\right) .
\end{aligned}
$$

The research for such kind of transformations is under current investigations. Algebraic transformations of hypergeometric functions of modular origin, are related to the monodromy of the underlying linear differential equations. This piece of research was started by the seminal contribution of Goursat, see [6] as the

Received 14 March 2018. Revised 23 April 2018.

${ }^{1}$ Corresponding Author

(C) 2018 Giovanni Mingari Scarpello and Daniele Ritelli. This is an open access article distributed under the Creative Commons Attribution License, which permits unrestricted use, distribution, and reproduction in any medium, provided the original work is properly cited. 
starting point, we highlight $[7,8,9]$ for some recent developments for this kind of approach. In [10] the theory of Theta functions is used to derive hypergeometric transformation formulæ, while [11] presents an approach referring to functional identities inspired by the work of [12]. Our contribution makes use of elementary methods, in the same spirit of [12].

In the first volume of his Traité [5], Legendre devotes more than a half of it to the elliptic integrals of first, second and third kind, showing their properties and explaining how to compute them by series, moduli transformation and so on. In Chapter XXVII, section II and III are dedicated to two integrals:

$$
R_{m}(k)=\int_{0}^{\frac{\pi}{2}} \frac{\mathrm{d} \varphi}{\sqrt[m]{1-k^{2} \sin ^{2} \varphi}}, \quad m=3,4 .
$$

First we provide a brief account of the Legendre's step in such computations, where he used plenty of the so-called Cauchy-Schlömich variable transformation, in order to evaluate definite integrals, see [13] for some historical references. Then we compute $R_{3}$ and $R_{4}$ using pure hypergeometric techniques obtaining new hypergeometric transformations for the Gauss function ${ }_{2} \mathrm{~F}_{1}$, see equations (4) and (13) below.

In the following we will use the integral representation theorem for the Gauss function ${ }_{2} \mathrm{~F}_{1}$

$$
{ }_{2} \mathrm{~F}_{1}\left(\begin{array}{c|c}
a ; b & x \\
c & x
\end{array}\right)=\frac{\Gamma(c)}{\Gamma(c-a) \Gamma(a)} \int_{0}^{1} \frac{u^{a-1}(1-u)^{c-a-1}}{(1-x u)^{b}} \mathrm{~d} u,
$$

which is traditionally ascribed to Euler, but really due to Legendre [14]. We will employ also the hypergeometric representation of the complete elliptic integral of first kind:

$$
\mathbf{K}(k)=\int_{0}^{1} \frac{\mathrm{d} u}{\sqrt{\left(1-u^{2}\right)\left(1-k^{2} u^{2}\right)}}=\frac{\pi}{2}{ }_{2} \mathrm{~F}_{1}\left(\begin{array}{c}
\frac{1}{2} ; \frac{1}{2} \\
1
\end{array} \mid k^{2}\right) .
$$

\section{Main results}

In this section we prove, using the evaluation of the integrals (3) for $k=4$ and $k=3$ given in [5], two hypergeometric transformation formulæ. Observe that the $k=4$ case is cited, without any quotation, by [15] entry 807.01 page 293, while there is no reference for $k=3$. Our first theorem comes from the case $k=4$ :

Theorem 2.1. If $0<b<1$ then:

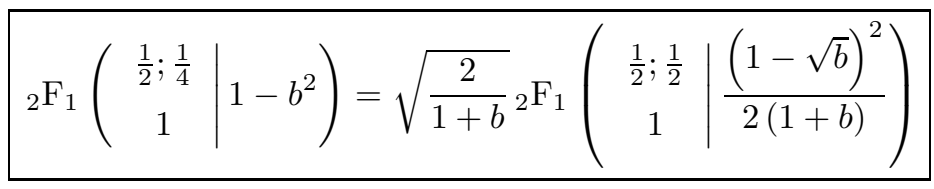


Proof. In order to proof (4) we first recall the way with Legendre computed the definite integral:

$$
R_{4}(c):=\int_{0}^{\frac{\pi}{2}} \frac{\mathrm{d} \varphi}{\sqrt[4]{1-c^{2} \sin ^{2} \varphi}}
$$

We start, choosing $b, c \in \mathbb{R}^{+}$such that $b^{2}+c^{2}=1$. Then change variable in (5):

$$
\frac{1-x^{4}}{c^{2}}=\sin ^{2} \varphi
$$

Observing that from (6) we get

$$
\cos ^{2} \varphi=\frac{x^{4}-b^{2}}{c^{2}}
$$

so that differentiating (6) we obtain:

$$
\frac{-4 x^{3}}{c^{2}} \mathrm{~d} x=2 \sin \varphi \cos \varphi \mathrm{d} \varphi=2 \frac{\sqrt{1-x^{4}}}{c} \frac{\sqrt{x^{4}-b^{2}}}{c} \mathrm{~d} \varphi
$$

which leads to our first integral transformation:

$$
R_{4}(c)=\int_{\sqrt{b}}^{1} \frac{2 x^{2}}{\sqrt{\left(1-x^{4}\right)\left(x^{4}-b^{2}\right)}} \mathrm{d} x .
$$

Then Legendre uses a Cauchy-Schlömich change of variable, putting:

$$
z=\frac{1}{2}\left(x^{2}+\frac{b}{x^{2}}\right) \text {. }
$$

Here the situation is similar to that, also studied by Legendre, which we presented in [3]. In fact, to use correctly the change of variable (7), since the transformation is not monotonic, we have to split the relevant integration domain in two sub-intervals, which are detected solving (7)

$$
x^{2}=z \pm \sqrt{z^{2}-b} .
$$

Looking at Figure 1 we infer that integral (5a) has to be evaluated splitting in two intervals $[\sqrt{b}, \sqrt[4]{b}]$ and $[\sqrt[4]{b}, 1]$, where of course in the first using (8) we have to choose the "-" sign, while in the second integral we have to take the "+" sign. Then using the denesting relation for quadratic surds, solving for $x$ in (8) we arrive at:

$$
x=\frac{1}{\sqrt{2}}(\sqrt{z+\sqrt{b}} \pm \sqrt{z-\sqrt{b}}) .
$$

For $x \in[\sqrt{b}, \sqrt[4]{b}]$ the integral transformation provides:

$$
\int_{\sqrt{b}}^{\sqrt[4]{b}} \frac{2 x^{2} \mathrm{~d} x}{\sqrt{\left(1-x^{4}\right)\left(x^{4}-b^{2}\right)}}=\frac{1}{2 \sqrt{2}} \int_{\sqrt{b}}^{\frac{1+b}{2}} \frac{\mathrm{d} z}{\sqrt{\left(\frac{1+b}{2}\right)^{2}-z^{2}}}\left(\frac{1}{\sqrt{z-\sqrt{b}}}-\frac{1}{\sqrt{z+\sqrt{b}}}\right) .
$$




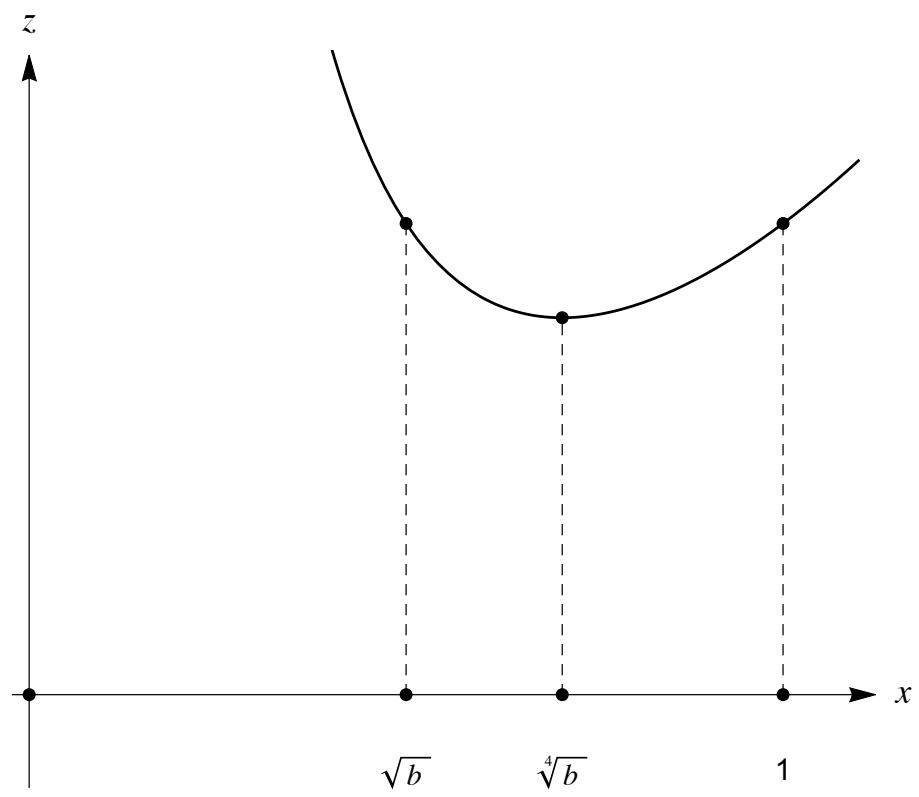

FiguRE 1. Cauchy-Schlömich transformation (7)

Similarly, for $x \in[\sqrt[4]{b}, 1]$ the integral transformation provides:

$$
\int_{\sqrt[4]{b}}^{1} \frac{2 x^{2} \mathrm{~d} x}{\sqrt{\left(1-x^{4}\right)\left(x^{4}-b^{2}\right)}}=\frac{1}{2 \sqrt{2}} \int_{\sqrt{b}}^{\frac{1+b}{2}} \frac{\mathrm{d} z}{\sqrt{\left(\frac{1+b}{2}\right)^{2}-z^{2}}}\left(\frac{1}{\sqrt{z-\sqrt{b}}}+\frac{1}{\sqrt{z+\sqrt{b}}}\right) .
$$

Therefore, summing, we can infer that

$$
R_{4}(c)=\frac{1}{\sqrt{2}} \int_{\sqrt{b}}^{\frac{1+b}{2}} \frac{\mathrm{d} z}{\sqrt{\left[\left(\frac{1+b}{2}\right)^{2}-z^{2}\right](z-\sqrt{b})}} .
$$

Now using entry 3.131-6 of [16] or (236.00) of [15] we obtain:

$$
R_{4}(c)=\sqrt{\frac{2}{1+b}} K\left(\frac{1-\sqrt{b}}{\sqrt{2(1+b)}}\right) .
$$

On the other side we can evaluate $R_{4}(c)$ in hypergeometric terms as:

$$
R_{4}(c)=\frac{\pi}{2}{ }_{2} \mathrm{~F}_{1}\left(\begin{array}{c|c}
\frac{1}{2} ; \frac{1}{4} & c^{2} \\
1 &
\end{array}\right)
$$


Now, comparing equation (11) and (10), representing the complete elliptic integral of first kind in terms of Gauss ${ }_{2} \mathrm{~F}_{1}$ function, we obtain (4), recalling relation $c^{2}+b^{2}=1$.

The second result comes from the evaluation of (3) when $k=3$.

Theorem 2.2. If $0<b<1$ and

$$
\alpha(b)=\frac{1}{2}-\frac{\sqrt{3}\left(b^{4}+2 b^{3}+2 b+1\right)}{4\left(b^{2}+b+1\right) \sqrt{b^{4}+b^{2}+1}}
$$

then the hypergeometric transformation formula holds true:

$$
{ }_{2} \mathrm{~F}_{1}\left(\begin{array}{c|c}
\frac{1}{2} ; \frac{1}{3} \\
1
\end{array} \mid 1-b^{6}\right)=\left(\frac{27}{\left(b^{2}+b+1\right)^{2}\left(b^{4}+b^{2}+1\right)}\right)^{1 / 4}{ }_{2} \mathrm{~F}_{1}\left(\begin{array}{c}
\frac{1}{2} ; \frac{1}{2} \\
1
\end{array} \mid \alpha(b)\right)
$$

Proof. Here we start from (3) taking $k=3$ and using for $R_{3}(c)$ the change of variable:

$$
\frac{1-x^{3}}{c^{2}}=\sin ^{2} \varphi
$$

which transforms the $R_{3}(c)$ integral into:

$$
R_{3}(c)=\frac{3}{2} \int_{\sqrt[3]{b^{2}}}^{1} \frac{x}{\sqrt{\left(1-x^{3}\right)\left(x^{3}-b^{2}\right)}} \mathrm{d} x=\frac{3}{2} \int_{n^{2}}^{1} \frac{x}{\sqrt{\left(1-x^{3}\right)\left(x^{3}-n^{6}\right)}} \mathrm{d} x
$$

where, to simplify the notation, we put $n^{3}=b$ being $0<n<1$. In fact, observing that from (14) follows:

$$
\cos ^{2} \varphi=\frac{x^{3}-b^{2}}{c^{2}}
$$

then, differentiating (14) we find

$$
\frac{-3 x^{2}}{c^{2}} \mathrm{~d} x=2 \sin \varphi \cos \varphi \mathrm{d} \varphi=2 \frac{\sqrt{1-x^{3}}}{c} \frac{\sqrt{x^{3}-b^{2}}}{c} \mathrm{~d} \varphi
$$

which shows (15). Legendre then uses again a Cauchy-Schlömich transformation

$$
z=x+\frac{n^{2}}{x}
$$

We repeat the same consideration to use correctly the change of variable (16): the lack of monotonicity requires again to split the interval of integration. Looking at Figure 2 we have to split the interval of integration in $\left[n^{2}, n\right]$ and $[n, 1]$, recall that $n<1$. To work with variable transformation (16) we revisit Legendre's path, providing a more rigorous approach, in order to manage properly the separation of the domain of integration. First write (16) as:

$$
x^{2}+n^{2}=x z
$$




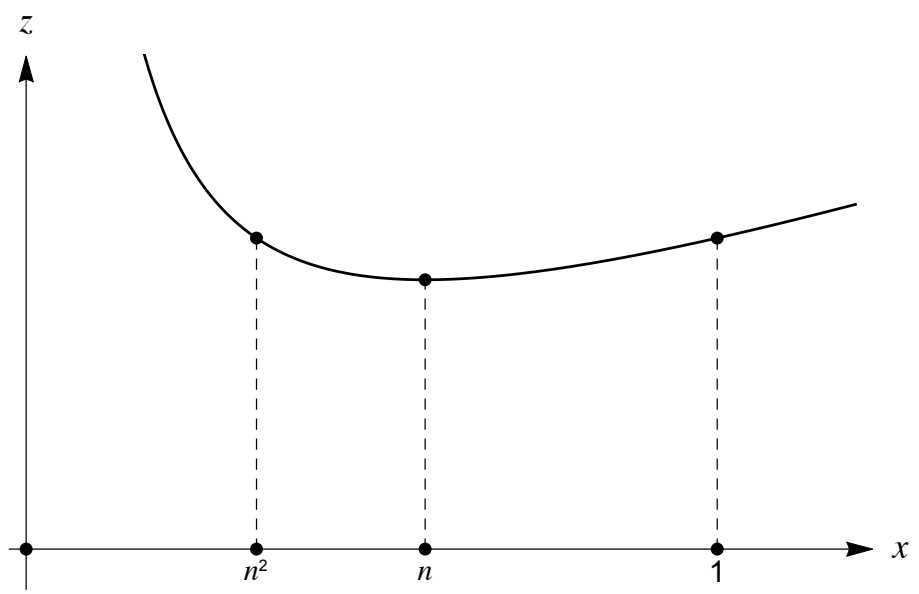

FIGURE 2. Cauchy-Schlömich transformation (16)

then using (17) compute

$$
\begin{aligned}
& (x+n)^{2}=x^{2}+n^{2}+2 n x=x z+2 n x=x(z+2 n), \\
& (x-n)^{2}=x^{2}+n^{2}-2 n x=x z-2 n x=x(z-2 n),
\end{aligned}
$$

For $x \in\left[n^{2}, n\right]$, since $x-n<0$ extracting square root in (18) we find:

$$
x+n=\sqrt{x} \sqrt{z+2 n}, \quad x-n=-\sqrt{x} \sqrt{z-2 n} .
$$

Summing side by side equations (19) we obtain:

$$
2 \sqrt{x}=\sqrt{z+2 n}-\sqrt{z-2 n} .
$$

We now use (20) to get the differential term of the change of variable (16), observe that since $x \in\left[n^{2}, n\right]$ the (16) transformation is decreasing, see Figure 2, therefore when $x \in\left[n^{2}, n\right]$ we have

$$
x^{-1 / 2} \mathrm{~d} x=\frac{1}{2}\left(\frac{1}{\sqrt{z-2 n}}-\frac{1}{\sqrt{z+2 n}}\right) \mathrm{d} z
$$

thus, observing that:

$$
\int_{n^{2}}^{n} \frac{x}{\sqrt{\left(1-x^{3}\right)\left(x^{3}-n^{6}\right)}} \mathrm{d} x=\int_{n^{2}}^{n} \frac{x^{3 / 2} x^{-1 / 2} \mathrm{~d} x}{\sqrt{\left(1-x^{3}\right)\left(x^{3}-n^{6}\right)}}
$$

and solving for $x$ in (16) always minding that $x \in\left[n^{2}, n\right]$ we get:

$$
x=\frac{1}{2}\left(z-\sqrt{z^{2}-4 n^{2}}\right) .
$$


Eventually, after heavy computations helped by computer algebra, we arrive at:

$$
x=\frac{1}{2}\left(z-\sqrt{z^{2}-4 n^{2}}\right) \Longrightarrow \frac{x^{3 / 2}}{\sqrt{\left(1-x^{3}\right)\left(x^{3}-n^{6}\right)}}=\frac{1}{\sqrt{1+n^{6}+3 n^{2} z-z^{3}}}
$$

In conclusion, using (21) and (23) we obtain:

$$
\begin{aligned}
& \int_{n^{2}}^{n} \frac{x}{\sqrt{\left(1-x^{3}\right)\left(x^{3}-n^{6}\right)}} \mathrm{d} x=\frac{1}{2} \int_{2 n}^{1+n^{2}} \\
& \times\left(\frac{1}{\sqrt{(z-2 n)\left(1+n^{6}+3 n^{2} z-z^{3}\right)}}-\frac{1}{\sqrt{(2 n+z)\left(1+n^{6}+3 n^{2} z-z^{3}\right)}}\right) \mathrm{d} z
\end{aligned}
$$

The argument for $x \in[n, 1]$ is similar, but the transformation of the integral in (21) is ruled by:

$$
x^{-1 / 2} \mathrm{~d} x=\frac{1}{2}\left(\frac{1}{\sqrt{z-2 n}}+\frac{1}{\sqrt{z+2 n}}\right) \mathrm{d} z
$$

which provides:

$$
\begin{aligned}
& \int_{n}^{1} \frac{x}{\sqrt{\left(1-x^{3}\right)\left(x^{3}-n^{6}\right)}} \mathrm{d} x=\frac{1}{2} \int_{2 n}^{1+n^{2}} \\
& \times\left(\frac{1}{\sqrt{(z-2 n)\left(1+n^{6}+3 n^{2} z-z^{3}\right)}}+\frac{1}{\sqrt{(2 n+z)\left(1+n^{6}+3 n^{2} z-z^{3}\right)}}\right) \mathrm{d} z
\end{aligned}
$$

Joining (24) and (25) we finally obtain

$$
\int_{n^{2}}^{1} \frac{x}{\sqrt{\left(1-x^{3}\right)\left(x^{3}-n^{6}\right)}} \mathrm{d} x=\int_{2 n}^{1+n^{2}} \frac{\mathrm{d} z}{\sqrt{(z-2 n)\left(1+n^{6}+3 n^{2} z-z^{3}\right)}}
$$

Now factoring we can write:

$$
1+n^{6}+3 n^{2} z-z^{3}=\left(n^{2}+1-z\right)\left(\left(z+\frac{n^{2}+1}{2}\right)^{2}+\left(\frac{\sqrt{3}\left(1-n^{2}\right)}{2}\right)^{2}\right)
$$

and then we use entry 259.00 page 133 of [15] to provide the left hand side of (26) in terms of complete elliptic integral of first kind:

$$
\int_{n^{2}}^{1} \frac{x}{\sqrt{\left(1-x^{3}\right)\left(x^{3}-n^{6}\right)}} \mathrm{d} x=\frac{2}{\sqrt[4]{3}\left(1+n+n^{2}\right)^{3 / 4}\left(1-n+n^{2}\right)^{1 / 4}} \boldsymbol{K}\left(k_{0}(n)\right)
$$

where

$$
k_{0}^{2}(n)=\frac{1}{2}-\frac{\sqrt{3}\left(n^{4}+2 n^{3}+2 n+1\right)}{4\left(n^{2}+n+1\right) \sqrt{n^{4}+n^{2}+1}}
$$


Thus, recalling (15), using (26b) and (26c) we obtain

$$
R_{3}(c)=\left(\frac{27}{\left(b^{2 / 3}-b^{1 / 3}+1\right)\left(b^{2 / 3}+b^{1 / 3}+1\right)^{3}}\right)^{1 / 4} \boldsymbol{K}(k(b))
$$

where

$$
k^{2}(b)=\frac{1}{2}-\frac{\sqrt{3}\left(b^{4 / 3}+2 b+2 b^{1 / 3}+1\right)}{4\left(b^{2 / 3}+b^{1 / 3}+1\right)\left(b^{4 / 3}+b^{2 / 3}+1\right)^{1 / 2}} .
$$

On the other side, we can evaluate $R_{3}(c)$ using the hypergeometric function:

$$
R_{3}(c)=\frac{\pi}{2}{ }_{2} \mathrm{~F}_{1}\left(\begin{array}{c|c}
\frac{1}{2} ; \frac{1}{3} & c^{2} \\
1 &
\end{array}\right) .
$$

Thesis (13) follows representing the complete elliptic integral of first kind in terms of Gauss ${ }_{2} \mathrm{~F}_{1}$ function, replacing $b$ with $b^{3}$ in (27) and (12).

\section{Conclusion}

Legendre in his Traité [5] was committed in searching identities among first kind complete elliptic integrals of different moduli, inspired by famous modular transformation:

$$
\mathbf{K}(k)=\frac{1}{1+k} \mathbf{K}\left(\frac{2 \sqrt{k}}{1+k}\right) .
$$

Searching for similar transformations he represented several kind of integrals in terms of complete elliptic integrals of first kind. Our contribution, starting from the elliptic evaluation of integrals $R_{3}(c)$ and $R_{4}(c)$, see (3), consists of the hypergeometric evaluation of such integrals, which leads to new, for the best of our knowledge, functional relations for the Gauss hypergeometric function, formulæ (4) and (13). A possible new research scenario consists of investigating further hypergeometric relations arising from integrals $R_{k}(c)$ when $k>4$.

\section{Competing Interests}

The author(s) do not have any competing interests in the manuscript.

\section{Acknowledgments}

The second author is supported by an Italian RFO research grant.

\section{REFERENCES}

1. Mingari Scarpello, G. , \& Ritelli, D. (2009). The hyperelliptic integrals and $\pi$. Journal of Number Theory, 129(12), 3094-3108.

2. Mingari Scarpello, G., \& Ritelli, D. (2011). $\pi$ and the hypergeometric functions of complex argument. Journal of Number Theory, 131, 1887-1900. 
3. Mingari Scarpello, G., \& Ritelli, D. (2014). Legendre hyperelliptic integrals, $\pi$ new formulae and Lauricella functions through the elliptic singular moduli. Journal of Number Theory, 135, 334-352.

4. Mingari Scarpello, G., \& Ritelli, D. (2014). On computing some special values of multivariate hypergeometric functions. Journal of Mathematical Analysis and Applications, $420(2), 1693-1718$.

5. Legendre, A. M. (1825), Traité des fonctions elliptiques et des intégrales Euleriennes. Tome premier, Imprimerie de Huzard-Courcier.

6. Goursat, E. (1881). Sur l'équation différentielle linéaire, qui admet pour intégrale la série hypergéométrique. Ann. Sci. Ecole Norm. Sup.(2), 10, 3-142.

7. Almkvist, G., Van Straten, D., \& Zudilin, W. (2011). Generalizations of Clausen's formula and algebraic transformations of CalabiYau differential equations. Proceedings of the Edinburgh Mathematical Society, 54(2), 273-295.

8. Maier, R. (2007). Algebraic hypergeometric transformations of modular origin. Transactions of the American Mathematical Society, 359(8), 3859-3885.

9. Vidūnas, R. (2009). Algebraic transformations of Gauss hypergeometric functions. Funkcialaj Ekvacioj, 52(2), 139-180.

10. Cooper, S., Ge, J., \& Ye, D. (2015). Hypergeometric transformation formulas of degrees 3, 7, 11 and 23. Journal of Mathematical Analysis and Applications, 421(2), 1358-1376.

11. Cooper, S., \& Zudilin, W. (2016). Hypergeometric modular equations. arXiv preprint arXiv:1609.07276.

12. Aycock, A. (2013). On proving some of Ramanujan's formulas for $\frac{1}{\pi}$ with an elementary method. arXiv preprint arXiv:1309.1140.

13. Amdeberhan, T., Glasser, M. L., Jones, M. C., Moll, V. H., Posey, R., \& Varela, D. (2010). The Cauchy-Schlömilch transformation. arXiv preprint arXiv:1004.2445.

14. Legendre, A.M., (1811), Exercices de Calcul Intégral sur Divers Ordres de Transcendantes et sur les Quadratures.Paris: Courcier. OCLC

15. Byrd, P. F., \& Friedman, M. D. (1971). Handbook of Elliptic Integrals for Engineers and Scienti 2nd Ed. Springer.

16. Gradshteyn, I. S., \& Ryzhik, I. M. (2014). Table of integrals, series, and products. Academic press.

\section{Giovanni Mingari Scarpello}

via Negroli, 6 Milan, Italy.

e-mail: giovannimingari@yahoo.it

Daniele Ritelli

Dipartimento di Scienze Statistiche, via Belle Arti, 41, 40126 Bologna Italy.

e-mail: daniele.ritelli@unibo.it 